\title{
Wireless power transfer with transmit diversity [version 1;
}

\section{peer review: 1 approved, 1 not approved]}

\author{
Yee-Loo Foo (ic)
}

Faculty of Engineering, Multimedia University, Cyberjaya, Selangor, 63100, Malaysia

V1 First published: $13 \operatorname{Sep} 2021,10: 916$

https://doi.org/10.12688/f1000research.72986.1

Latest published: 13 Sep 2021, 10:916

https://doi.org/10.12688/f1000research.72986.1

\section{Abstract}

Background: Wireless power transfer is important for energizing and recharging the Internet-of-Things (IoT) cordlessly. Harnessing energy effectively from radio waves has become a crucial task. It is known that diversities at the transmitting antenna and waves (i.e. simultaneous continuous waves with center frequencies separated apart) can enhance the radio frequency (RF) to direct current (DC) energy conversion. What remains unknown is the extent of which the wave diversity enhances the conversion gain. This study attempts to examine the RF-to-DC conversion gain of applying wave diversity. This paper investigates the effects of wave diversity on the energy conversion efficiency, and contributes the analytical expression that relate the conversion efficiency to the diversity count, i.e. the number of simultaneously transmitted sinewaves.

Methods: We adopted a theoretical approach to the problem. First, we derived and presented a theoretical model that incorporated different forms of transmit diversity, i.e. antenna and wave diversities. This model then connected a RF-to-DC energy conversion model resulting from polynomial fitting on circuit simulation results. With the availability of these two models, we determined the theoretical energy conversion gain of simultaneously transmitting multiple sinewaves. Results: The results showed that transmitting multiple sinewaves simultaneously yields diversity gain and higher energy conversion efficiency. Most importantly, the gain and conversion efficiency can now be theoretically quantified. For example, at certain RF power measured at the receiver circuit, the diversity gain of transmitting four sinewaves is 2.6 (as compared to transmitting single sinewave). In fact, both the diversity gain and conversion efficiency increased with the number of simultaneously transmitted sinewaves. In another example, the conversion efficiency of transmitting four sinewaves is 0.1 as compared to 0.075 of two sinewaves.

Conclusions: In summary, this paper presents a novel analytical expression for wave diversity in the context of wireless power transfer.

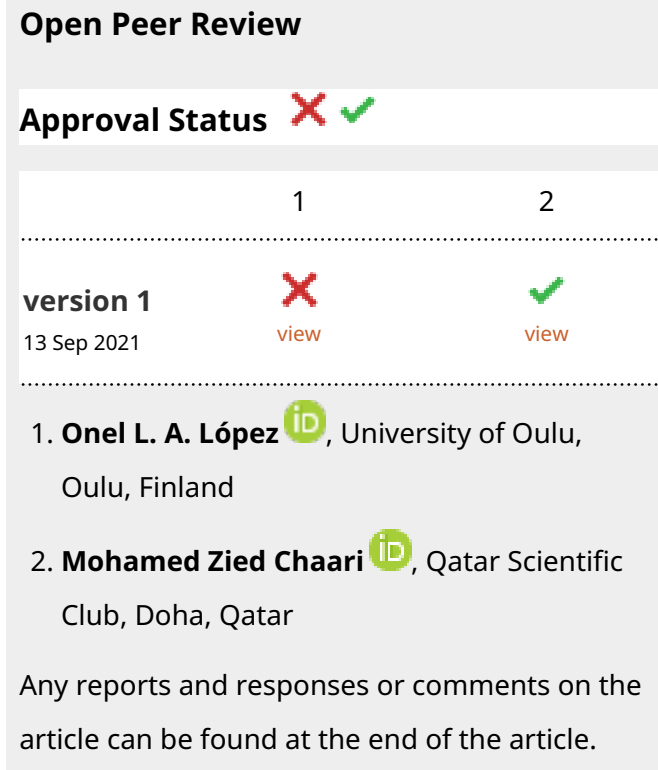


Keywords

wireless power transfer, energy harvesting, RF-to-DC energy

conversion, transmit diversity, antenna diversity, Internet-of-Things

(IoT)

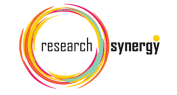

This article is included in the Research Synergy

Foundation gateway.

Corresponding author: Yee-Loo Foo (ylfoo.mmu@gmail.com)

Author roles: Foo YL: Conceptualization, Data Curation, Formal Analysis, Funding Acquisition, Investigation, Methodology, Project Administration, Resources, Software, Validation, Visualization, Writing - Original Draft Preparation, Writing - Review \& Editing

Competing interests: No competing interests were disclosed.

Grant information: Multimedia University IR Fund 2020/16310, Project "Reliable and Resilient Internet-of-Things (IoT) Networks", Principal Investigator: Yee-Loo Foo.

The funders had no role in study design, data collection and analysis, decision to publish, or preparation of the manuscript.

Copyright: $\odot 2021$ Foo YL. This is an open access article distributed under the terms of the Creative Commons Attribution License, which permits unrestricted use, distribution, and reproduction in any medium, provided the original work is properly cited.

How to cite this article: Foo YL. Wireless power transfer with transmit diversity [version 1; peer review: 1 approved, 1 not approved] F1000Research 2021, 10:916 https://doi.org/10.12688/f1000research.72986.1

First published: 13 Sep 2021, 10:916 https://doi.org/10.12688/f1000research.72986.1 


\section{Introduction}

The Internet-of-Things (IoT) have become omnipresent nowadays, bringing to us some new challenges. To build a robust and resilient IoT network is one of the main challenges. The IoT communication infrastructure must be reliable and trustable. The IoT networks must be resilient against broken links and power outages. Nevertheless, replacing and recharging the device batteries are not only troublesome and impractical, but also nearly impossible in some cases. Wireless power transfer is a solution to this problem. ${ }^{1-3}$ It can energize and recharge the IoT devices cordlessly. Hence, harnessing energy effectively from radio waves becomes a very important task. ${ }^{2}$ Figure 1 depicts the concept of wireless power transfer. Wireless energy harvesting is possible even over fading and shadowing channels. ${ }^{4,5}$ Chen et al. ${ }^{4}$ have formulated the cumulative distribution function (CDF) and outage probability of the harvested power under these circumstances. On the other hand, Clerckx and $\mathrm{Kim}^{5}$ have assessed the effect of fading on the radio frequency-to-direct current (RF-to-DC) energy conversion, and revealed that fading actually enhanced the conversion efficiency. There are numerous ways of enhancing radio wave energy harvesting, e.g. via transmission over multiple antennas, transmitting multiple sinewaves of different frequencies simultaneously, transmitting specially designed waveforms, etc. ${ }^{5-7} \mathrm{Khan}$ et al. ${ }^{6}$ have expressed the efficiency of wireless power transfer in terms of the number of transmit antennas. Clerckx and Bayguzina $^{7}$ have studied the design of energy waveforms that enhanced wireless power transfer to a rectenna, which comprised of an antenna and a diode rectifier. The design involved specifying the amplitudes and phases of the multisinewaves in response to the channel condition. The fluctuation in the waveform excited the rectenna circuit. The maximum load current in the circuit was expressed as a function of the numbers of transmit antennas and sinewaves. ${ }^{7}$ Clerckx and $\mathrm{Kim}^{5}$ have furthered the study based on the similar diode rectenna model, and in addition, a curve-fitting model. Constructed from the data collected from simulations, the curve-fitting model is simpler, resulting in simpler and more tractable analyses. Clerckx and $\mathrm{Kim}^{5}$ evaluated the antenna and phase diversities that resulted in channel fluctuations, similar effect as of fading. They proved that even without using carefully designed energy waveform, which required channel state information (CSI), simple antenna diversity or phase diversity of multi-sinewaves were effective for wireless power transfer. Their findings were supported by the results of experimenting with prototypes. Based on the curve-fitting model, transmitting multi-sinewaves with center frequencies that were equally spaced out did enhance the RF-to-DC conversion efficiency, ${ }^{5}$ but an analytical quantification on the attained gain remains absent. This study aims to examine the RF-to-DC conversion gain with respect to the wave diversity, and contributes the relevant analysis that expresses the energy conversion efficiency as a function of the antenna diversity and 'wave diversity', i.e. the number of transmitted sinewaves.

In the next section, we describe the communication system model that incorporates transmit diversity. This is followed by the RF-to-DC energy conversion model. Based on the models of transmit diversity and energy conversion, we produce the relevant results and show them in the Results section. This is followed by a Discussion section. Finally, we present the conclusions in the last section.

\section{Methods}

Ethical approval

This work has met the research ethics requirement and received the university Research Ethics Committee's approval, with the number EA1772021.

\section{Section 1: Transmit diversity model}

A sender transmits signal $x(t)$, a continuous wave (CW) that has been modulated by symbol $s(t)$, on $M$ antennas at time $t$. The transmit signal on antenna $m$ is varied by phase $\varphi_{m}(t)$, i.e.

$$
x_{m}(t)=\sqrt{\frac{2 P}{M}} \Re\left\{s(t) e^{j\left[\omega_{0} t+\varphi_{m}(t)\right]}\right\}
$$

$\omega_{0}$ is the carrier frequency. $E\left[|s(t)|^{2}\right]=1$. After propagating over the wireless channel, the signal picked up at single receive antenna is

$$
y(t)=\sqrt{\frac{2 P}{M \Lambda}} \mathfrak{R}\left\{h(t) s(t) e^{j \omega_{0} t}\right\}
$$

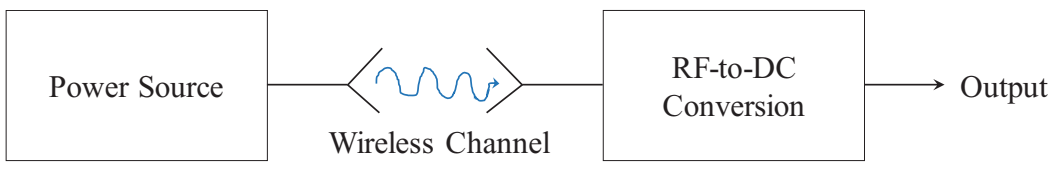

Figure 1. Wireless power transfer. 
$\Lambda$ is the path loss, $h(t)$ represents the overall time-varying channel gain.

$$
h(t)=\sum_{m=1}^{M} h_{m} e^{j \varphi_{m}(t)}
$$

$h_{m}$ is the amplitude gain from antenna $m$ due to channel fading. Let $r(t)=h(t) s(t)$.

$$
y(t)=\sqrt{\frac{2 P}{M \Lambda}} \mathfrak{R}\left\{r(t) e^{j \omega_{0} t}\right\}
$$

The effective RF power level at the input of an energy harvester is

$$
P_{\mathrm{RF}}=E\left[y^{2}(t)\right]
$$

Assume that $\left\{\varphi_{m}(t)\right\}_{\forall m}$ are uniformly distributed over $2 \pi$ and are independent, and $h_{m}=1 \forall m$. It can be shown that

$$
P_{\mathrm{RF}}=\bar{P}_{\mathrm{RF}} \frac{|r(t)|^{2}}{M}
$$

$|r(t)|^{2}$ is the received signal envelope.

$$
\bar{P}_{\mathrm{RF}}=E\left[P_{\mathrm{RF}}\right]=\frac{P}{\Lambda}
$$

Given certain expression for $s(t)$, we can determinel $r(t)$ land thus $P_{\mathrm{RF}}$. In the following, we consider a combination of multiple sinewaves of which the center frequencies are constantly separated apart. Suppose the multisine waveforms are given by

$$
s(t)=\sum_{n=0}^{N-1} \frac{1}{\sqrt{N}} \exp \left(j n \Delta_{w} t\right), \Delta_{w}=2 \pi \Delta_{f}
$$

$\Delta_{w}$ is the inter-carrier frequency spacing. Assume that $\left\{\varphi_{m}(t)\right\}_{\forall m}$ are uniformly distributed over $2 \pi$ and are independent, and $h_{m}=1 \forall m$.

$$
r(t)=\sum_{m=1}^{M} \sum_{n=0}^{N-1} \frac{1}{\sqrt{N}} e^{j n \Delta_{w} t} e^{j \varphi_{m}(t)}
$$

Consider a special case of $M=1$.

$$
r(t)=\frac{1}{\sqrt{N}} \sum_{n=0}^{N-1} e^{j\left[n \Delta_{w} t+\varphi(t)\right]}
$$

To calculate $|r(t)|$, let us first consider a simple case of $N=2$ sinewaves where $n=1,2$.

$$
r(t)=\frac{1}{\sqrt{2}}\left(e^{j\left[\Delta_{1} t+\varphi(t)\right]}+e^{j\left[\Delta_{2} t+\varphi(t)\right]}\right), \Delta_{2}=2 \Delta_{1}
$$

It can be shown that

$$
|r(t)|^{2}=1+\cos \theta
$$

where

$$
\theta=\theta(t)=\Delta_{1} t, 0 \leq \theta<2 \pi
$$


When $M=1$ and $N=3$, we find that

$$
|r(t)|^{2}=1+\frac{2}{3}(2 \cos \theta+\cos 2 \theta)
$$

By induction, it can be shown that for $M=1$,

$$
|r(t)|^{2}=1+\frac{2}{N}[(N-1) \cos \theta+(N-2) \cos 2 \theta+\ldots+2 \cos (N-2) \theta+\cos (N-1) \theta]
$$

To the best of our knowledge, (14) is a novel expression.

Section 2: RF-to-DC energy conversion

Based on a simulation study on a rectenna circuit and curve fitting on the collected data, Clerckx and Kim ${ }^{5}$ have shown that the RF-to-DC energy conversion can be represented by a polynomial as follows:

$$
\ln P_{\mathrm{DC}}=u\left(\ln P_{\mathrm{RF}}\right)^{2}+v \ln P_{\mathrm{RF}}+w
$$

Based on the results from the circuit simulations conducted by Clerckx and Kim, ${ }^{5}$ when the input was a CW, the coefficients $u, v$, and $w$ of the polynomial took the following values:

$$
\begin{aligned}
& u=-0.0669, v=-0.1317, w=-6.3801 \\
& \text { for }-40 \mathrm{dBm} \leq P_{\mathrm{RF}} \leq-5 \mathrm{dBm}
\end{aligned}
$$

Since $P_{\mathrm{RF}}=\bar{P}_{\mathrm{RF}} \frac{|r(t)|^{2}}{M}$,

$$
\ln P_{\mathrm{DC}}=u\left[\ln \left(\bar{P}_{\mathrm{RF}} \frac{|r(t)|^{2}}{M}\right)\right]^{2}+v \ln \left(\bar{P}_{\mathrm{RF}} \frac{|r(t)|^{2}}{M}\right)+w
$$

After some workings, we have the following expression:

$$
\ln P_{\mathrm{DC}}=\ln P_{\mathrm{DC}, 0}+\left(\ln \frac{|r(t)|^{2}}{M}\right)\left[u\left(\ln \frac{|r(t)|^{2}}{M}+2 \ln \bar{P}_{\mathrm{RF}}\right)+v\right]
$$

where

$$
\ln P_{\mathrm{DC}, 0}=u\left(\ln \bar{P}_{\mathrm{RF}}\right)^{2}+v \ln \bar{P}_{\mathrm{RF}}+w
$$

$P_{\mathrm{DC}, 0}$ is the DC power harvested from $\bar{P}_{\mathrm{RF}}$. Eq. (17) can also be expressed in the following form:

$$
P_{\mathrm{DC}}=P_{\mathrm{DC}, 0} \cdot e_{\mathrm{td}}
$$

$e_{\mathrm{td}}$ is the gain from transmit diversity,

$$
e_{\mathrm{td}}=\left(\frac{|r(t)|^{2}}{M}\right)^{u\left(\ln \frac{\left.\operatorname{lr}(t)\right|^{2}}{M}+2 \ln \bar{P}_{\mathrm{RF}}\right)+v}
$$

The average harvested DC power,

$$
\bar{P}_{\mathrm{DC}}=E\left[P_{\mathrm{DC}}\right]=P_{\mathrm{DC}, 0} \cdot \bar{e}_{\mathrm{td}}
$$

where

$$
\bar{e}_{\mathrm{td}}=E\left[e_{\mathrm{td}}\right]=E\left[\left(\frac{|r(t)|^{2}}{M}\right)^{u\left(\ln \frac{|\mathrm{r}(t)|^{2}}{M}+2 \ln \bar{P}_{\mathrm{RF}}\right)+v}\right]
$$


The resultant RF-to-DC conversion efficiency is given by

$$
\varepsilon_{0}=\frac{P_{\mathrm{DC}, 0}}{\bar{P}_{\mathrm{RF}}}
$$

Substituting (23) into (21),

$$
\bar{P}_{\mathrm{DC}}=\bar{P}_{\mathrm{RF}} \varepsilon_{0} \bar{e}_{\mathrm{td}}
$$

The average RF-to-DC conversion efficiency is thus given by

$$
\varepsilon_{\mathrm{avg}}=\frac{\bar{P}_{\mathrm{DC}}}{\bar{P}_{\mathrm{RF}}}=\varepsilon_{0} \bar{e}_{\mathrm{td}}
$$

\section{Results}

The following Figures 2 and 3 present the novel results.

The positive result ${ }^{8}$ of using multiple sinewaves is demonstrated in the transmit diversity gain (Figure 2) and the actual resultant energy conversion efficiency (Figure 3). From Figures 2 and 3, we see that larger number of sinewaves, with center frequencies being equally spaced out, results in higher RF-to-DC energy conversion, despite using only single transmit antenna $(M=1)$. This is especially true at low RF input power to the rectenna circuit. For example, at $\bar{P}_{\mathrm{RF}}=10^{-7} \mathrm{~W}$, the transmit diversity gain is 2.6 (of 4 sinewaves) as compared to 1.5 (of 2 sinewaves). Nevertheless, the gain starts to diminish as the RF input power becomes higher, as revealed in Figure 2.

Figure 3 shows similar trend of which higher number of sinewaves results in higher conversion efficiency. For example, at $\bar{P}_{\mathrm{RF}}=3.2 \times 10^{-6} \mathrm{~W}$, the conversion efficiency is 0.1 (of 4 sinewaves) as compared to 0.075 (of 2 sinewaves).

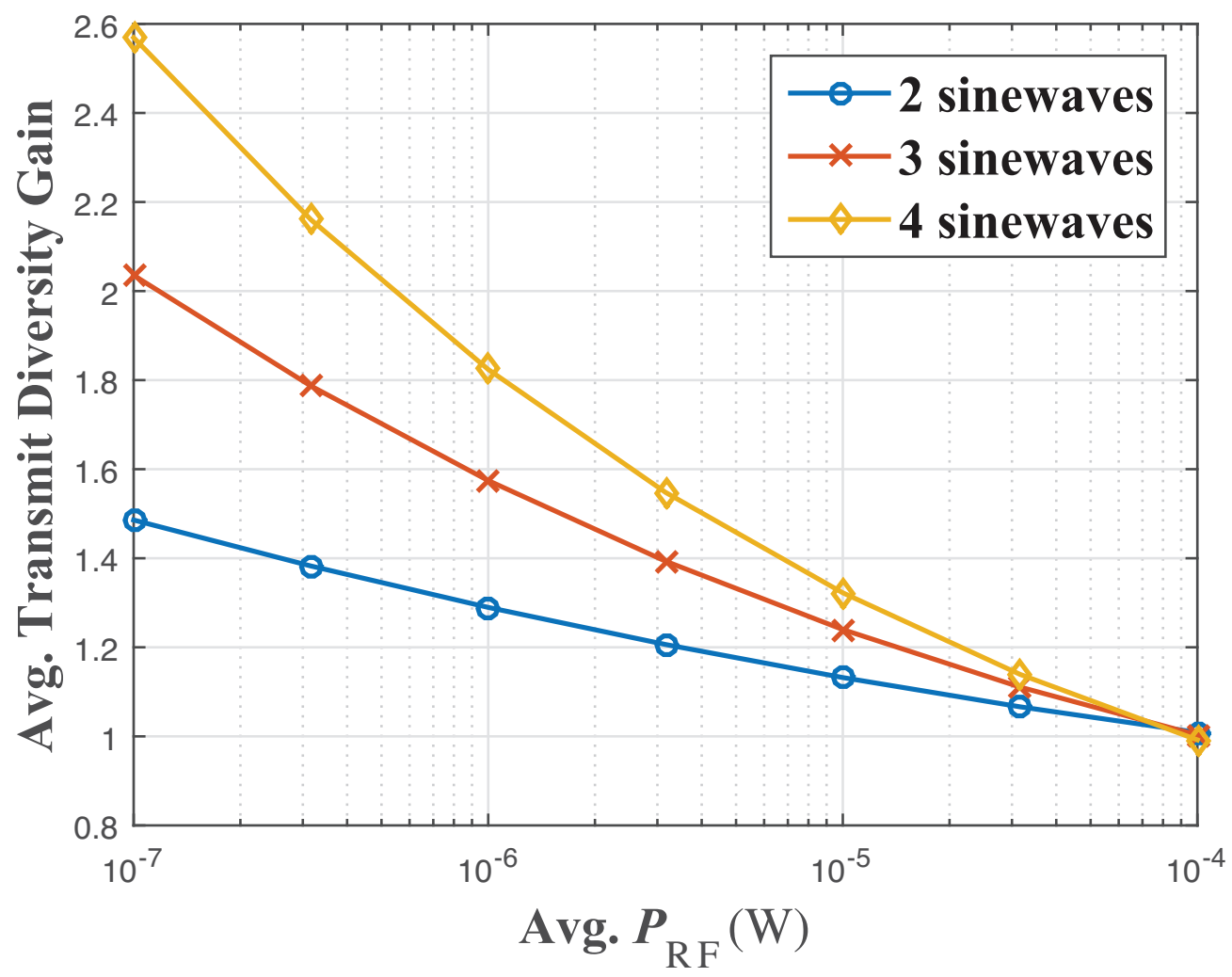

Figure 2. $\overline{\boldsymbol{e}}_{\mathrm{td}}$ Vs. $\overline{\boldsymbol{P}}_{\mathrm{RF}}$ for multiple sinewaves. 


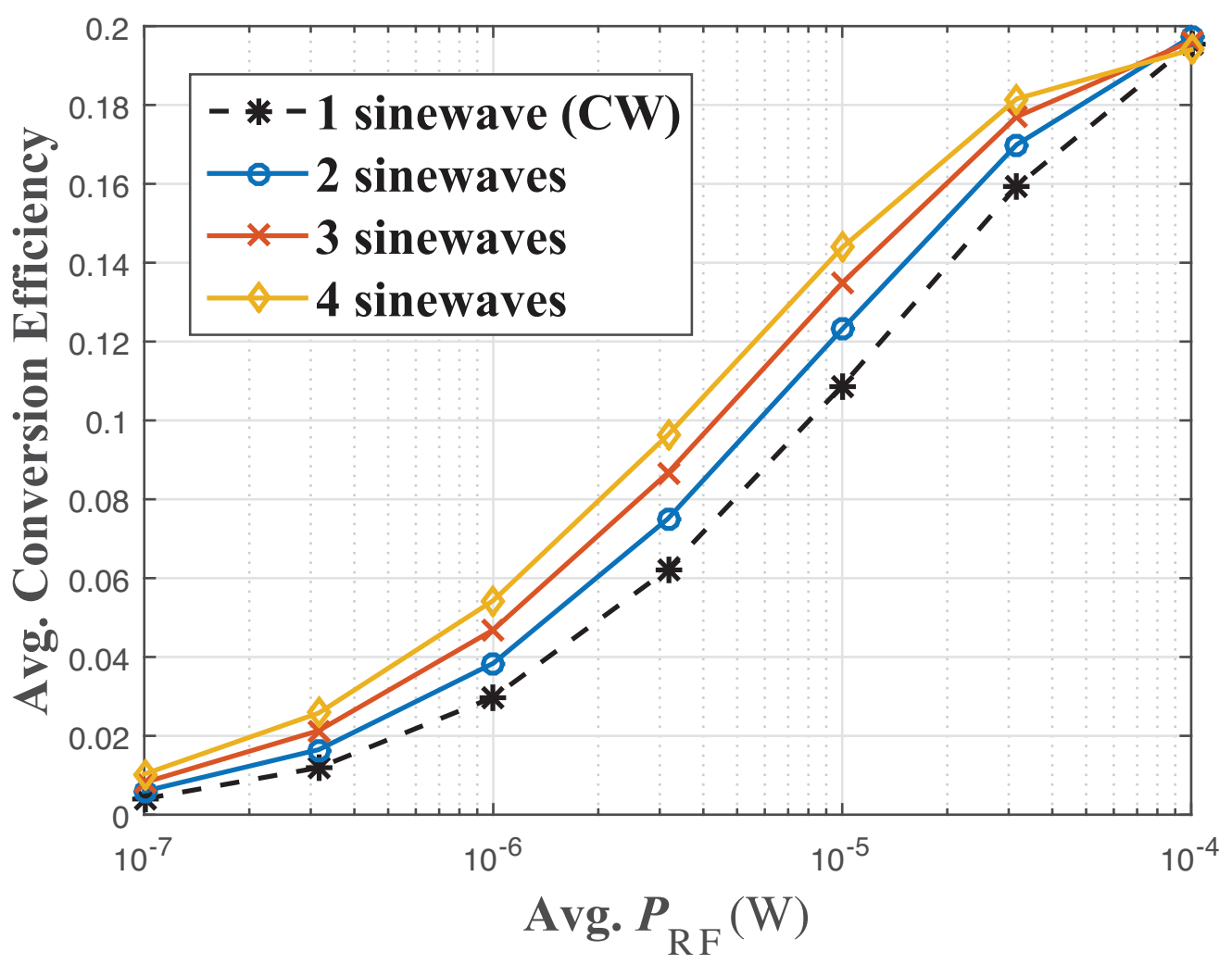

Figure 3. $\varepsilon_{\mathrm{avg}}$ vs. $\overline{\boldsymbol{P}}_{\mathrm{RF}}$ for multiple sinewaves.

\section{Discussion}

In this section, we discuss the impacts of the results. By experimenting with prototypes, Clerckx and $\mathrm{Kim}^{5}$ had proven that transmitting multi-sinewaves with center frequencies that were equally spaced out could enhance the RF-to-DC conversion efficiency. Nevertheless, an analytical expression that relates the attained gain to the wave diversity remains absent. The analysis presented in this paper not only fills this gap, but may also benefit the experiments of wireless power transfer that are based on hardware, e.g. using a microwave transmitter to wirelessly power up a LED. ${ }^{3}$ They can experiment with the antenna diversity and wave diversity that have been theoretically proven effective.

Nevertheless, the analysis presented here has not considered the separation distance between the $M$ transmitting antennas. Taking into account of this factor could provide a more comprehensive analysis on the effects of beamforming. ${ }^{1,2}$ This is especially important in the context of massive wireless power transfer, ${ }^{1}$ where a large number of IoT devices are targeted.

\section{Conclusions}

Wireless power transfer benefits from transmit diversity, be it antenna diversity or wave diversity. The objective of this paper is to express the RF-to-DC energy conversion efficiency as a function of the transmit diversity. Such a mathematical expression was not available previously. This paper contributes an analytical expression that relates the diversity gain and conversion efficiency to the number of simultaneous sinewaves with center frequencies that are equally spaced. Our next step is to investigate the wave diversity gain of beamforming antennas.

\section{Author contributions}

Y.-L. Foo designs the research, constructs the computer program, conducts the experiment, reports and discusses the findings.

Data availability

https://doi.org/10.17605/OSF.IO/K9ZNU

Open Science Framework: Wireless Power Transfer, https://doi.org/10.17605/OSF.IO/39SJV. ${ }^{8}$ 
The project contains the following underlying data:

\section{- Data.txt}

Data are available under the terms of the Creative Commons Attribution 4.0 International license (CC-BY 4.0).

References

1. Lopez OLA, Alves H, Souza RD, et al.: Massive Wireless Energy Transfer: Enabling Sustainable IoT Toward 6G Era. IEEE Internet Things. 2021; 8(11): 8816-8835.

Publisher Full Text

2. Molefi M, Markus ED, Abu-Mahfouz A: Wireless Power Transfer for IoT Devices-A Review Proc. - 2019 Int. Multidiscip. Inf. Technol. Eng. Conf. IMITEC 2019. 2019; vol. i.

Publisher Full Text

3. Chaari MZ, Al-Maadeed S: Wireless Power Transmission for the Internet of Things (IoT). 2020 IEEE Int. Conf. Informatics, IoT, Enabling Technol. ICIOT 2020. 2020; pp. 549-554.

Publisher Full Text

4. Chen $\mathrm{Y}$, Zhao N, Alouini MS: Wireless Energy Harvesting Using Signals from Multiple Fading Channels. IEEE Trans. Commun. Nov. 2017; 65(11): 5027-5039.

Publisher Full Text
5. Clerckx B, Kim J: On the Beneficial Roles of Fading and Transmit Diversity in Wireless Power Transfer with Nonlinear Energy Harvesting. IEEE Trans. Wirel. Commun. Nov. 2018; 17(11): 7731-7743.

Publisher Full Text

6. Khan TA, Yazdan A, Heath RW: Optimization of power transfer efficiency and energy efficiency for wireless-powered systems with massive MIMO. IEEE Trans. Wirel. Commun. Nov. 2018; 17(11): 7159-7172.

Publisher Full Text

7. Clerckx B, Bayguzina E: Waveform Design for Wireless Power Transfer. IEEE Trans. Signal Process. Dec. 2016; 64(23): 6313-6328.

Publisher Full Text

8. Foo Y-L: Wireless Power Transfer. Open Science Framework. Publisher Full Text 


\section{Open Peer Review}

\section{Current Peer Review Status:}

\section{Version 1}

Reviewer Report 28 October 2021

\section{https://doi.org/10.5256/f1000research.76603.r96552}

(C) 2021 Chaari M. This is an open access peer review report distributed under the terms of the Creative Commons Attribution License, which permits unrestricted use, distribution, and reproduction in any medium, provided the original work is properly cited. The author(s) is/are employees of the US Government and therefore domestic copyright protection in USA does not apply to this work. The work may be protected under the copyright laws of other jurisdictions when used in those jurisdictions.

\section{Mohamed Zied Chaari}

Fabrication Lab, Qatar Scientific Club, Doha, Qatar

In general, the structure of the manuscript is well organized, the methods are well described, and the procedures are well conducted. The results are sound and the conclusions are based on the experimental data.

Comments on the manuscript:

The abstract must be rewritten. An abstract is a 150- to 250-word paragraph that provides readers with a quick overview of your essay or report and its organization. It should express the wireless power transmission and key points and suggest any implications or applications of the research you discuss in the paper.

The structure is general idea/reason; aim and goals; materials; methods and procedures; main results; general conclusion.

Good quality, high-resolution figures should be provided.

I have a suggestion to include references 1 and 2 in your work, in order to strengthen the content. The author is working on wireless power transfer with transmit diversity, and these citations give him more idea about our work and can use them for upgrading his work and make a good overview of the WPT.

\section{References}

1. Chaari M, Al-Maadeed S: Increase the Efficiency of IoT Devices by Using the Wireless Power Transmission in the Industrial Revolution 4.0. International Journal of Online and Biomedical Engineering (iJOE). 2021; 17 (07). Publisher Full Text

2. Chaari MZ, Al-madeed S: Wireless Power Transmission for the Internet of Things (IoT). 2020 IEEE International Conference on Informatics, IoT, and Enabling Technologies. 2020. Publisher Full Text 
Is the work clearly and accurately presented and does it cite the current literature? Yes

Is the study design appropriate and is the work technically sound?

Yes

Are sufficient details of methods and analysis provided to allow replication by others?

Yes

If applicable, is the statistical analysis and its interpretation appropriate?

Partly

Are all the source data underlying the results available to ensure full reproducibility?

Yes

Are the conclusions drawn adequately supported by the results?

Partly

Competing Interests: Harvesting CFL light for charging a supercapacitor.

Reviewer Expertise: microwave technology, RF harvesting energy, and wireless power charging.

I confirm that I have read this submission and believe that I have an appropriate level of expertise to confirm that it is of an acceptable scientific standard.

Reviewer Report 21 September 2021

https://doi.org/10.5256/f1000research.76603.r94148

(C) 2021 López 0. This is an open access peer review report distributed under the terms of the Creative Commons Attribution License, which permits unrestricted use, distribution, and reproduction in any medium, provided the original work is properly cited.

\section{Onel L. A. López}

Centre for Wireless Communications, University of Oulu, Oulu, Finland

The author investigates an interesting research problem and system setting. The aim is at quantifying the diversity gain from multi sinusoidal transmissions, i.e., wave diversity. My major comments are as follows:

The main contribution of this work seems to be related to Equation (14). If author adopted a proof by induction, this needs to be properly realized. Please write Equation (14) in a more compact form using summation symbol. Also, the derivation of Equation (14) is only for the case of $M=1$, what about a general, more practical, case of $M>1$ ?

Why are the introduced phase shifts considered uniformly distributed? This means there is 
no CSI at the transmitter, or at least it is not exploited. Given that there is vast literature on CSI-free wireless power transfer in the last years, I recommend motivating this assumption better.

Since the conversion efficiency depends on $|r(t)|^{2}$, it would be interesting to see the latter in a plot as a function of $\mathrm{N}$. Currently Section 2 seems completely disconnected from Section 1. What is the main idea behind the discussions/derivations in Section 2?

Numerical results are not currently included for multi-antenna setups.

Other minor comments:

Proofreading is needed. Specifically, adjust Abstract to be in present tense. Correct typos, and grammar issues along the paper.

The definition of $P$ is missing in Equation (1). After Equation (1), how is $y(t)$ given as a function of $x_{m}(t)$ ?

Is the expectation in Equation (4) taken with respect to both waveform and fading randomness? Clarify.

Is the work clearly and accurately presented and does it cite the current literature? Partly

Is the study design appropriate and is the work technically sound?

No

Are sufficient details of methods and analysis provided to allow replication by others? Yes

If applicable, is the statistical analysis and its interpretation appropriate? Partly

Are all the source data underlying the results available to ensure full reproducibility? No source data required

Are the conclusions drawn adequately supported by the results? Partly

Competing Interests: No competing interests were disclosed.

Reviewer Expertise: Wireless communications, Wireless power transfer, Energy harvesting, Internet of Things, Positioning systems, Cellular systems

I confirm that I have read this submission and believe that I have an appropriate level of expertise to state that I do not consider it to be of an acceptable scientific standard, for reasons outlined above. 
The benefits of publishing with F1000Research:

- Your article is published within days, with no editorial bias

- You can publish traditional articles, null/negative results, case reports, data notes and more

- The peer review process is transparent and collaborative

- Your article is indexed in PubMed after passing peer review

- Dedicated customer support at every stage

For pre-submission enquiries, contact research@f1000.com 